\title{
New possible source of huge neutrino bursts
}

\author{
S. E. Juralevich and V.V.Tikhomirov * \\ Institute for Nuclear Problems, Belarussian State University, Bobruiskaya 11, 220050 Minsk, Belarus
}

(Dated: June 20, 2012)

\begin{abstract}
It is shown that primordial black holes ( $\mathrm{PBHs}$ ) of masses $M \geq 5 \times 10^{14} \mathrm{~g}$ are able to absorb white dwarfs (WD) completely for the time of their existence, giving rise to formation of black holes of WD mases. The WD absorption is accompanied by up to $10^{52}$ erg neutrino bursts which could both considerably contribute to the cosmic neutrino flux and open up a new way of PBHs detection, especially effective in placing new constraints on abundance of nonevaporating $\mathrm{PBHs}$ with masses $M \gg 10^{15} \mathrm{~g}$.
\end{abstract}

PBHs could have formed in the early universe [1, 2] from initial inhomogeneities, topological defects (strings, bubbles) or at cosmological phase transitions. PBHs provide a unique probe of the Big Bang and even their nonexistence gives vital cosmological information [3, [4, 5].

The Hawking radiation [6] allows to set up severe constraints on the $\mathrm{PBH}$ abundance. In particular, the radiation of all the evaporated PBHs could have given its contribution to the diffuse cosmic flux [7, 8, 9]. Very stringent bound has been obtained from the absence of a detectable diffuse flux of $100 \mathrm{MeV}$ photons [7]. It was claimed, however, that these bounds can be improved using available data on diffuse neutrino flux [8, 9].

Another way of PBHs search consists in attempts to detect gamma [7] or neutrino [8] bursts accompanying the final stage of PBH evaporation. The PBHs which complete their evaporation at present have had the initial mass $M_{0}$ equal to the critical one (Hawking mass) $M_{*} \simeq 5 \times 10^{14} \mathrm{~g}$. For all the time of their evaporation such PBHs could emit about $M_{*} c^{2} \simeq 5 \times 10^{35} \mathrm{erg}$, where $c$ is the speed of light, what is much less, for example, than the energy of supernova explosion reaching $3 \times 10^{53} \mathrm{erg}$. The comparatively low energy release undoubtedly constrains the search possibilities of the most intensively evaporating $\mathrm{PBHs}$ and makes utterly ineffective that of weakly evaporating PBHs with $M \simeq M_{0} \gg M_{*}$, the constraints on which are more than ten orders lower than that on PBHs with $M_{0} \simeq M_{*}$ [3, 目, 司.

We would like to suggest here an alternative method of PBHs search, free of this deficiency. Namely, we will show that being trapped inside a white dwarf (WD) a PBH of mass $M_{0} \geq M_{*}$ can completely absorb it initiating a huge neutrino burst with the energy reaching $10^{52} \mathrm{erg}$.

First, we will consider the simplest case of nonrotating cool WD and apply the general relativistic theory of spherical, steady-state, adiabatic accretion of continuous Pascal liquid [10, 11] to show that the time of a complete WD absorption by a Schwarzschild PBH can be less than the age of the universe. A special consideration has shown that the heating caused by PBH Hawking radiation, WD matter compression and neutronization does not violate the high degeneracy of WD matter. The mass-energy density $E$ and pressure $P$ of the degenerate WD matter are given by the equations [10, 11, 12]

$$
\begin{gathered}
E(n)=\mu c^{2} n+\frac{m_{e}^{4} c^{5}}{8 \pi^{2} \hbar^{3}}\left[x\left(2 x^{2}+1\right) \sqrt{1+x^{2}}\right. \\
-\operatorname{Arsh} x]-\frac{3}{4} \alpha^{\prime} m_{e}^{2} c^{3} x, \\
P(n)=n \frac{d E}{d n}-E(n),
\end{gathered}
$$

where $n$ is the electron number density,

$$
x=x(n)=\frac{p_{F}(n)}{m_{e} c}
$$

is the electron Fermi momentum $p_{F}(n)=\left(3 \pi^{2} n\right)^{1 / 3} \hbar$ expressed in $m_{e} c$ units, $m_{e}$ and $\hbar$ are the electron mass and the Planck constant, respectively. $\mu$ is the WD matter mass which falls at one electron. We will consider the most widespread case of carbon WDs for which $\mu \simeq 2 m_{n}$, where $m_{n}$ is the nucleon mass. The term of Eq.(1) containing the small parameter

$$
\alpha^{\prime}=\frac{4}{5}\left(\frac{3}{2 \pi}\right)^{1 / 3} \alpha Z^{2 / 3} \ll 1,
$$

where $\alpha \simeq 1 / 137$ and $Z$ is the atomic number, describes the Coulomb correction [10] which is principally important in the case of low WD density.

The spherical, steady-state accretion flow of degenerate Fermi gas is described by the radial component of 4 -velocity $u$ and the electron number density $n$. Both of them depend only on the radial Schwarzschild coordinate measured from the PBH center and obey [10, 11] the electron conservation equation

$$
\dot{M}=4 \pi \mu n(r) c u(r) r^{2}=\mathrm{const}
$$

which gives the rest mass accretion rate as well as the relativistic Bernoulli equation

$$
\begin{aligned}
& \left(\frac{E(n)+P(n)}{\mu c^{2} n}\right)^{2}\left(1+u^{2}-\frac{r_{g}}{r}\right) \\
& =\left(\frac{E\left(n_{0}\right)+P\left(n_{0}\right)}{\mu c^{2} n_{0}}\right)^{2}=\text { const. }
\end{aligned}
$$


containing the gravitational radius $r_{g}=2 G M / c^{2} \simeq$ $1.5\left(M / 10^{15} g\right) 10^{-15} \mathrm{~m}$ where $G$ is the Newton's constant. The steady-state accretion assumes that $n \rightarrow n_{0}=$ const at $r \rightarrow \infty$. The constant signs in Eqs. (5) and (6) reflect the independence on $r$.

The basic point of the theory [10, 11] consists in existence of a special (sonic) point $r=r_{s}$ at which the relations

$$
u_{s}^{2}=u^{2}\left(r_{s}\right)=\frac{a_{s}^{2}}{1+3 a_{s}^{2}}=\frac{r_{g}}{4 r_{s}}
$$

hold where $a_{s}=a\left(r_{s}\right)$ is the sound speed $a=(d P / d E)^{1 / 2}$ expressed in $c$ units and taken at $r=r_{s}$. Using the Emden solution 10, 12 for the WD density distribution one can make sure that, if a PBH with mass $M$ is situated at the WD center, the uniformity condition in the essential accretion region $r \sim r_{s}$ is fulfilled at $M \leq 10^{-3} M_{W D}$ where $M_{W D}$ is the WD mass. The electron number density $n_{0}$ in Eq. (6) can be considered as that at the WD center in this case.

Substituting Eqs. (1), (2) and (7) into Eq. (6) one obtains in the first order with respect to $m_{e} / \mu \simeq 2.74 \times$ $10^{-4}$ the equation

$$
\frac{2+x_{s}^{2}}{\sqrt{1+x_{s}^{2}}}-\alpha^{\prime} x_{s}=2\left(\sqrt{1+x_{0}^{2}}-\alpha^{\prime} x_{0}\right)
$$

connecting $x_{s}=x\left(n\left(r_{s}\right)\right)$ with $x_{0}=x\left(n_{0}\right)$. Eq. (8) demonstrates that the Coulomb correction is indeed essential at the density $\rho=E / \mathrm{c}^{2} \leq 100 \mathrm{~g} / \mathrm{cm}^{3}$ giving rise to the existence of two solutions of Eq. (8) at $x_{0}<2 \alpha^{\prime}$ $\left(\alpha^{\prime} \simeq 0.015\right.$ at $\left.Z=6\right)$. However here we will consider the usual case of much denser WDs in which $x_{0} \gg 2 \alpha^{\prime}$ and Eq. (8) has a single solution taking the simple form

$$
x_{s} \simeq 2 x_{0}+\frac{1}{4\left(1-\alpha^{\prime}\right) x_{0}}+O\left(\frac{1}{x_{0}^{2}}\right)
$$

or $\rho\left(r_{s}\right) \simeq 8 \rho_{0}$ in the ultrarelativistic limit of $x_{0} \gg 1$, $\rho_{0} \gg 2 \times 10^{6} \mathrm{~g} / \mathrm{cm}^{3}$. Eqs. (5) and (6) also allow one to calculate the parameter (3) maximum value

$$
x_{\max }=x\left(r_{g}\right) \simeq \frac{\left(1+x_{s}^{2}\right)^{1 / 4}}{2^{4 / 3}}\left(\frac{3 \mu}{m_{e}}\right)^{1 / 2} \simeq 42\left(1+x_{s}^{2}\right)^{1 / 4}
$$

at $r=r_{g}$. The corresponding Fermi energy $\varepsilon_{F}\left(r_{g}\right) \simeq$ $m_{e} c x\left(r_{g}\right)$ reaches $20-100 \mathrm{MeV}$.

Substituting $x_{s}$ into Eq. (7) one finds the values $n\left(r_{s}\right)$, $r_{s}$ and $u\left(r_{s}\right)$ allowing to calculate the right hand side of Eq. (5) and to obtain the law

$$
M(t)=\frac{M_{0}}{1-t / T_{a b s}}
$$

of the $\mathrm{PBH}$ accretion mass growth starting from its initial value $M_{0}=M(0)$ and characterized by the time

$$
\begin{aligned}
T_{a b s}= & \frac{\pi \hbar^{3}}{\sqrt{3} G^{2} \mu^{5 / 2} m_{e}^{3 / 2} M_{0}}\left(\frac{1}{\sqrt{1+x_{s}^{2}}}-\frac{\alpha^{\prime}}{x_{s}}\right)^{3 / 2} \\
& =\frac{27}{\left(M_{0} / 10^{15} g\right)}\left(\frac{1}{\sqrt{1+x_{s}^{2}}}-\frac{\alpha^{\prime}}{x_{s}}\right)^{3 / 2} G y r .
\end{aligned}
$$

which can be considered as that of WD absorption. It should be emphasized that though Eq. (11) loses its applicability at $M(t) \geq 10^{-3} M_{W D}$, the accuracy of the estimate (12) is quite high since Eq. (11) does not describe only a very small part $\Delta t / T_{a b s}=M_{0} /\left(10^{-3} M_{W D}\right)$ $\left(10^{-16}\right.$-th part at $\left.M_{0} \sim M_{*}\right)$ of the time (12). Thus, the approach [10, 11] has led us to a very precise estimate of the time of WD absorption by a PBH.

Using the relation (8) of $x_{s}$ with $x_{0}=x\left(n_{0}\right)$ one can calculate the time (12) dependence on the WD central density $\rho_{0}=E\left(n_{0}\right) / c^{2}$. Fig. 1 demonstrates that a PBH with initial mass $M_{0}=10^{15} \mathrm{~g}$ can absorb relativistic $\left(\rho_{0} \geq 10^{6} \mathrm{~g} / \mathrm{cm}^{3}\right)$ WDs for several Gyrs. Since $T_{a b s} \propto M_{0}^{-1}$, PBHs with initial masses $M_{0} \geq 10^{17} \mathrm{~g}$ will absorb WDs of any density for the time of their existence. The absorption process could give rise to formation of black holes of WDs masses which can not form in the star collapse process. Since WDs are quite abundant their absorption by $\mathrm{PBHs}$ could, in principle, be more productive source of star-mass black holes than the star collapse.

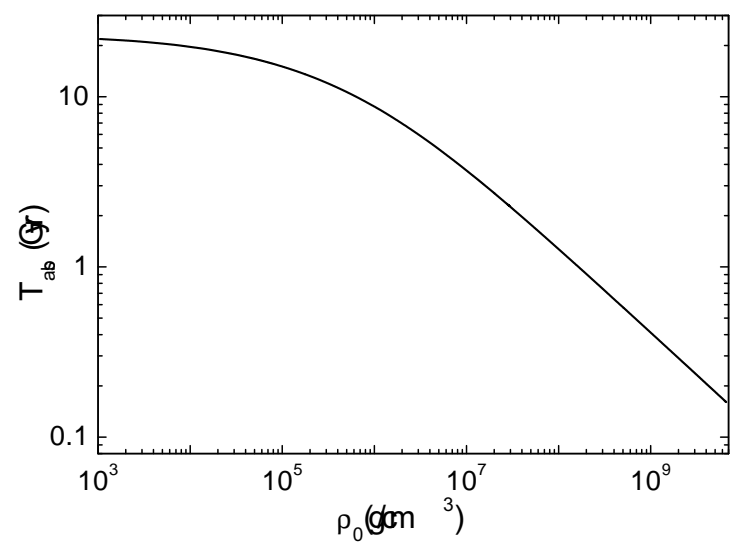

FIG. 1: Time of a complete WD absorption by a PBH with mass $M_{0}=10^{15} \mathrm{~g}$ as a function of WD central density.

A WD absorption by a PBH would be observed as its sudden disappearance accompanied by a moderate light $(x$-ray) flash. Such a disappearance, however, is difficult to observe at distances exceeding $100 \mathrm{ps}$. We will show now that a WD absorption by a PBH is accompanied by a huge neutrino burst detectable at much longer distances.

Neutrinos will be produced in neutronization reactions ${ }^{12} C+e^{-} \rightarrow{ }^{12} B+\nu,{ }^{12} B+e^{-} \rightarrow{ }^{12} B e+\nu$ in carbon WDs [10]) in the region with electron Fermi energy $\varepsilon_{F}(r)=$ $m_{e} c^{2} \sqrt{1+x^{2}(r)}$ exceeding the neutronization threshold energy $\Delta_{\mathrm{Z}}\left(\Delta_{\mathrm{C}}=13.88 \mathrm{MeV}, \Delta_{\mathrm{B}}=12.17 \mathrm{MeV}\right)$. Since $\varepsilon_{F}\left(r_{g}\right)$ exceeds $\Delta_{\mathrm{C}}$ considerably at any $x_{0}$ and $\rho_{0}$ (see Eqs. (8) and (10)) a PBH will give rise to the neutronization process in any carbon WD.

Both the local neutronization probability of a nucleus with atomic number $Z$ [10

$$
\Gamma_{\mathrm{Z}}=\Gamma_{\mathrm{Z}}\left(\varepsilon_{F}(r)\right)=\frac{\ln 2}{T_{\mathrm{Z}-1}} \frac{f\left(\Delta_{\mathrm{Z}}, \varepsilon_{F}\right)}{f\left(m_{e} c^{2}, \Delta_{\mathrm{Z}}\right)},
$$


where

$$
\begin{array}{r}
f\left(\varepsilon_{1}, \varepsilon_{2}\right)=\int_{\varepsilon_{1}}^{\varepsilon_{2}} f^{\prime} d \varepsilon, \\
f^{\prime}=\varepsilon\left(\varepsilon^{2}-m_{e}^{2} c^{4}\right)^{1 / 2}\left(\varepsilon-\Delta_{z}\right)^{2},
\end{array}
$$

and the accompanying neutrino emission intensity

$$
\begin{aligned}
I_{\nu}^{Z}=I_{\nu}^{Z}\left(\varepsilon_{F}(r)\right) & =\frac{\ln 2}{T_{\mathrm{Z}-1} f\left(m_{e} c^{2}, \Delta_{\mathrm{Z}}\right)} \\
& \times \int_{\Delta_{\mathrm{Z}}}^{\varepsilon_{F}}\left(\varepsilon-\Delta_{\mathrm{Z}}\right) f^{\prime} d \varepsilon .
\end{aligned}
$$

are determined by the local electron number density $n(r)$, the threshold energy $\Delta_{\mathrm{z}}$ and the half-life $T_{\mathrm{z}-1}$ of the daughter nucleus $\left(T_{\mathrm{B}}=20.20 \mathrm{~ms}, T_{\mathrm{Be}}=21.31 \mathrm{~ms}\right)$.

Neutronization begins at the "neutronization radius" $r_{n}>r_{g}$ at which $\varepsilon_{F}\left(n\left(r_{n}\right)\right)=\Delta_{\mathrm{C}}$ and $\rho_{n}=E\left(n\left(r_{n}\right)\right) / c^{2}$ $=3.90 \times 10^{10} \mathrm{~g} / \mathrm{cm}^{3}$ [10]. According to Eq. (9) one has $r_{n}=r_{s}$ at the central WD density $\rho_{0} \simeq \rho_{n} / 8 \simeq 4.90 \times$ $10^{9} \mathrm{~g} / \mathrm{cm}^{3}$. Since most of WDs have much smaller ones let us assume that $r_{n} \ll r_{s}$. The WD matter motion in the neutronization region $r_{g}<r<r_{n}$ is determined in this case rather by gravity than by pressure and is well described by the free fall equation

$$
u(r)=\sqrt{r_{g} / r}
$$

written in Lagrangian coordinates. Eq. (16) allows both to introduce a proper time $\tau(r)=\int_{r}^{r_{n}} d r /[c u(r)], \tau\left(r_{n}\right)=$ 0 , of elementary volume motion from $r_{n}$ to $r$ and to write the electron number conservation equation $r^{2} n u=$ const, which holds in neglect of electron absorption, in the form

$$
x(r)=x_{\max } \sqrt{r_{g} / r} .
$$

Eqs. (8) and (10) obtained in the model of steady-state accretion [10, 11] fail to describe the accretion rate saturation accompanied by the most intense neutrino emission. The last comes from the region $r \sim r_{\max }$ giving the main contribution to the integral determining the WD mass. According to the Emden solution for ultrarelativistic Fermi gas [10, 12] $r_{\max }$ equals $0.237 R_{W D}$, where $R_{W D}$ is the WD radius. The WD matter with the initial density $\rho\left(r_{\max }\right)=0.312 \rho_{0}$ experiences the attraction of the mass $0.37 M_{W D}$. Though this mass is contracted in the process of absorption by a $\mathrm{PBH}$ it determines the constant value of $r_{g}$ in Eqs. (16) and (17) describing the final stage of accretion accompanied by neutronization. The nonuniformity of the region $r \sim r_{\max }$ giving the main contribution to the neutrino emission will be taken into consideration by using $\rho\left(r_{\max }\right)$ instead of $\rho_{0}$ in Eqs. (8) and (10) determining the $x_{\max }$ value.

Let us introduce the $C, B$ and $B e$ nuclei fractions normalized by the equality $c_{\mathrm{C}}+c_{\mathrm{B}}+c_{\mathrm{Be}}=1$ and satisfying the initial conditions $c_{\mathrm{C}}(0)=1, c_{\mathrm{B}}(0)=c_{\mathrm{Be}}(0)=0$ at the moment $\tau\left(r_{n}\right)=0$ of passage through the neutronization

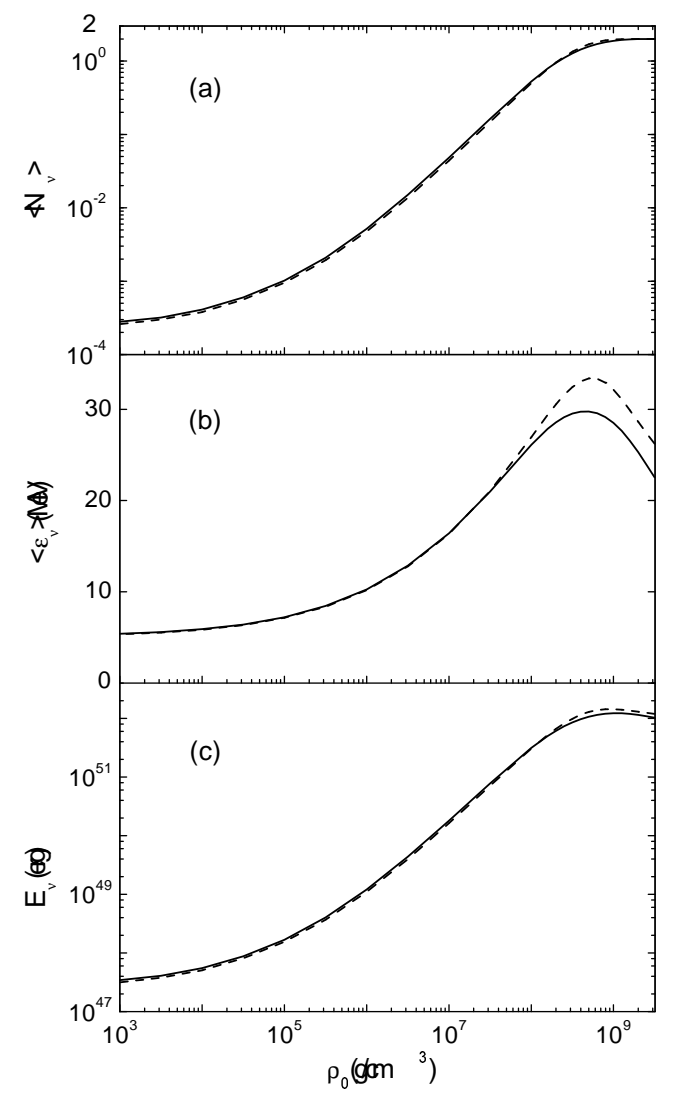

FIG. 2: Characteristics of neutrino emission process accompanying a carbon WD absorption by a $\mathrm{PBH}$ : (a) average neutrino number which falls at one nucleus, (b) average neutrino energy and (c) neutrino burst energy as a function of central WD density as predicted by the model described in the text (dashed line) and by a more sophisticated approach (full lines).

radius. The first two fractions obey the equations

$$
\begin{array}{r}
d c_{\mathrm{C}} / d \tau=-\Gamma_{\mathrm{C}} c_{\mathrm{C}} \\
d c_{\mathrm{B}} / d \tau=\Gamma_{\mathrm{C}} c_{\mathrm{C}}-\Gamma_{\mathrm{B}} c_{\mathrm{B}} .
\end{array}
$$

Eqs. (16) and (17) allow to pass to the variable $r$ in Eqs. (13) and (18) and easily integrate the last yielding the analytical expressions for $c_{\mathrm{C}}(r)$ and $c_{\mathrm{B}}(r)$, as well as for both the average neutrino number

$$
<N_{\nu}>=2-2 c_{\mathrm{C}}\left(r_{g}\right)-c_{\mathrm{B}}\left(r_{g}\right)
$$

which falls at one carbon nucleus and the average neutrino energy

$$
\begin{array}{r}
<\varepsilon_{\nu}>=\frac{1}{<N_{\nu}>} \int_{r_{g}}^{r_{n}}\left[I_{\nu}^{\mathrm{C}}(r) c_{\mathrm{C}}(r)\right. \\
\left.+I_{\nu}^{\mathrm{B}}(r) c_{\mathrm{B}}(r)\right] \frac{d r}{c u(r)} .
\end{array}
$$


The $\left\langle N_{\nu}>\right.$ and $\left\langle\varepsilon_{\nu}>\right.$ dependencies on the central WD density $\rho_{0}$ are given, respectively, on Figs. 2(a) and (b) along with the same dependencies evaluated using a more sophisticated model taking into account the electron absorption, the pressure influence on accreting matter motion and the nonequilibrium heating of the last which accompanies the neutronization process [13]. The negligible difference in predictions of these two models justifies the undertaken consideration of the simpler one.

Fig. 2(a) demonstrates the possibility of complete carbon and subsequent boron neutronization. The average and maximum neutrino energies can reach 30 (see Fig. 2(b)) and $100 \mathrm{MeV}$ (see Eqs. (9) and (10)), respectively. The dependence of the energy

$$
E_{\nu}=\frac{M_{W D}}{Z \mu}<N_{\nu}><\varepsilon_{\nu}>
$$

of the neutrino burst accompanying the total WD absorption on the central WD density is given on Fig. 2(c) demonstrating that $E_{\nu}$ can exceed $10^{52} \mathrm{erg}$. The neutrino burst duration will not considerably exceed the free fall time, since neutrino will travel through the WD matter nearly freely. The huge neutrino flux, nevertheless, will heat the WD matter up to $T \geq 10^{7} K$ and, possibly, initiate the nuclear burning [14] in the most dense WDs. These processes will be considered elsewhere.

Thus, we have demonstrated that the energy of a purely neutrino burst accompanying a WD absorption by a PBH is only a few orders less than that of a neutrinoantineutrino burst accompanying a supernova explosion [15]. If such a burst occurs at the typical distance of $10 k p s$ it will cause up to 50 events of $\nu e$ scattering in the Super-Kamiokande detector and, thus, can be detected with confidence. In fact, it looks feasible to design a detector able to detect most of the predicted neutrino bursts in our Galaxy 8 , 15. A possible frequency of these bursts depends on such uncertain factors as $\mathrm{PBH}$ abundance and mass distribution as well as on the probability of PBHs presence in WDs or capture by them. Taking into consideration the high WDs abundance in the Galaxy and assuming more or less uniform distribution of the moments of WDs complete absorption in time, optimistically one can hypothesize that the predicted neutrino bursts can be detected nearly every year.

According to Ref. [9] PBHs with masses $M_{0} \ll M_{*}$ contribute mostly to the diffuse neutrino flux, the study of which already allows to set up restrictions on the power of the "blue" spectrum of initial density fluctuations. The possibility of intensive neutrino emission by WDs being absorbed by $\mathrm{PBHs}$ radically change this picture. First, since the energy (21) 11-16 orders exceeds the rest energy $M_{*} c^{2}$, even a small number of PBHs absorbing most weakly rotating WDs can improve the bounds on PBHs absence by many orders. Second, since the heavier PBHs absorb WDs more readily, the diffuse neutrino flux, in fact, allows to set up the most stringent bounds on the nonevaporating $\mathrm{PBHs}$ with masses $M_{0} \gg M_{*}$.

Thus, we have demonstrated that PBHs can absorb WDs for the time of their existence giving rise to formation of black holes of WD's masses. The WDs absorption is accompanied by the neutrino bursts which can be detected directly and could have given a considerable contribution to the diffuse neutrino flux. Most considerably these neutrino bursts widen the possibilities of search of nonevaporating PBHs with masses $M_{0} \gg 10^{15} \mathrm{~g}$.

The authors would like to thank Professor M. Ju. Khlopov and the members of his seminar for valuable discussion and Professor V. G. Baryshevsky for support of our work.
[1] J.B. Zel'dovich and I.D. Novikov, Astron. J. (USSR) 43, 758 (1966).

[2] S.W. Hawking, Mon. Not. R. Astron. Soc. 152, 75 (1972).

[3] B.J. Carr, Astron. J. 206, 8 (1976); astro-ph/0102390.

[4] M.Ya. Khlopov, Cosmoparticle Physics (World Sci., Singapore, 1999).

[5] I.D. Novikov and V.P. Frolov, Physics of Black Holes (Kluwer, Dordrecht, 1989).

[6] S.W. Hawking, Nature 240, 30 (1974); D.N. Page, Phys. Rev. D 13, 1 (1976).

[7] B.J. Carr and I.H. MacGibbon, Phys. Repts. 307, 141 (1988).

[8] F. Halzen and B. Keszthelyi, hep-ph/9502268.
[9] E.V. Bugaev and K.V. Konishchev, astro-ph/0103265.

[10] S. Shapiro and S. Teukolsky, Black Holes, White Dwarfs and Neutron Stars (Wiley, New York, 1983).

[11] F. Michel, Astrophys. Space Sci. 15, 153 (1972).

[12] L.D. Landau and E.M. Lifshitz, Statistical physics (Pergamon, Oxford, 1980).

[13] G.S. Bysnovaty-Kogan and Z.F. Seidov, Astron. J. (USSR) 47, 139 (1970).

[14] S.S. Gerstein et al., Zh. Eksp. Teor. Fiz. 69, 5 (1975).

[15] H.V. Klapdor-Kleingrothaus and K. Zuber, Teilchenastrophysik (Teubner, Stutgart, 1997). 